\title{
Zum Nachweis der Standsicherheit in der tiefen Gleitfuge
}

\author{
Wolfgang Fellin
}

6.2.2017

This is the peer reviewed version of the following article: Fellin, Wolfgang (2017): Zum Nachweis der Standsicherheit in der tiefen Gleitfuge. geotechnik 40(3):162-171., which has been published in final form at https://doi.org/10.1002/gete.201600023. This article may be used for non-commercial purposes in accordance with Wiley Terms and Conditions for Use of Self-Archived Versions.

Der Nachweis der Standsicherheit in der tiefen Gleitfuge ist einer der Standardnachweise für eine verankerte Baugrubenwand. Nach einigem hin und her ist man derzeit wieder bei einer Sicherheitsdefinition angelangt, die der ursprünglichen Idee von Kranz entspricht. In diesem Beitrag wird gezeigt, warum dieser Nachweis an einigen Stellen mechanisch diskussionswürdig ist. Deshalb wird angeregt, einen direkten Nachweis über die Beanspruchung und den Widerstand in der Gleitfuge zu führen, welcher konform mit den anderen geotechnischen Nachweisen des Nachweisverfahrens 2 des Eurocodes ist. Für ein Berechnungsbeispiel ergeben sich mit dem hier vorgeschlagenen Nachweis ähnliche Ankerlängen wie bei einem Nachweis nach Kranz.

\section{Einleitung}

Die Modellbildung, also das Vereinfachen der komplexen Realität hin zu einem berechenbaren mechanischen Modell, gehört zu den Kernkompetenzen von Ingenieurinnen und Ingenieuren. In der Geotechnik ist dies generell schwierig, da es sich fast ausschließlich um dreidimensionale kontinuumsmechanische Aufgabenstellungen handelt. Das heißt, es müssen sehr oft einschneidende Vereinfachungen vorgenommen werden. Diese sollten aber gut begründbar sein, ansonsten können sie nicht vermittelt werden, und die daraus resultierenden Modelle werden dann in der Praxis mangels Verständnis als Black-Box verwendet. Das kann zu groben Anwendungensfehlern führen.

Der derzeit verwendete Nachweis der Standsicherheit in der tiefen Gleitfuge passt nicht zu den sonstigen Nachweisen des Eurocodes. Wo die Pro- bleme liegen, und wie der Nachweis sehr einfach in Übereinstimmung mit anderen Nachweisen gebracht wird, ist Thema des folgenden Textes.

\section{Mechanische Modellierung}

\subsection{Bruchmechanismus}

Bei der Dimensionierung eines verankerten Baugrubenverbaus wird zunächst nachgewiesen, dass das Erdwiderlager nicht versagt. Ein Versagen kann dann nur mehr durch eine Verdrehung des Verbaus erfolgen. Weiters wird nachgewiesen, dass die aus der Statik folgende Ankerkraft über die Verpressstrecke des Ankers eingeleitet werden kann, der Anker also nicht herausgezogen wird. Damit muss sich das Material zwischen Anker und Verbau bei einem möglichen Versagen des Gesamtsystems mit dem Anker bewegen. So- 
mit ist neben einem gesamtem Böschungsbruch (z.B. Gleitkreis außerhalb der verankerten Zone) nur noch der Versagenstyp der sogenannten tiefen Gleitfuge möglich, bei dem eine Verdrehung des Verbaus unter Ausbildung von Scherzonen wie z.B. in dem Experiment von Walz [18] in Bild 1 eintritt. Der Versagenszustand der nicht zwangsweise geführten Wand wurde in diesem Versuch durch kurzzeitiges Herabsetzen der Scherfestigkeit des Bodens bei Erschütterungen herbeigeführt (leichte Schläge mit einem Gummihammer auf den Rahmen des Modell-Behälters). Die zwischen Fußpunkt des Verbaus und Ankerlage leicht nach oben gekrümmte Scherzone wurde bereits von Jelinek u. Ostermayer [7] in Experimenten beobachtet, und wird als tiefe Gleitfuge (oder tiefe Gleitfläche) bezeichnet.

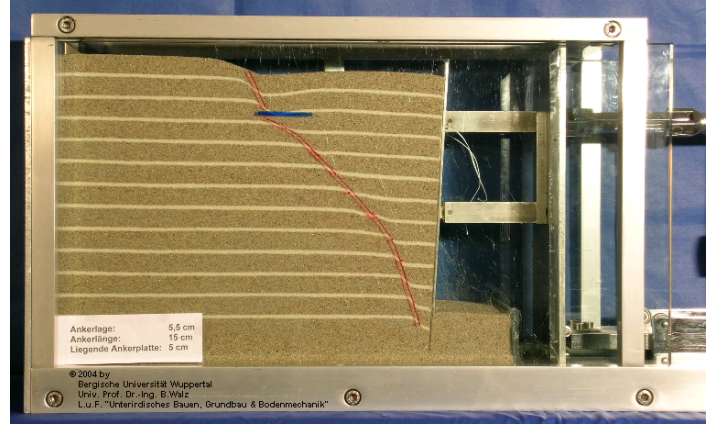

Bild 1: Tiefe Gleitfuge bei liegender Ankerplatte, aus [18. Die Scherzonen sind durch rote Doppellinien markiert.

Goldscheider [4] zeigt, dass die Bewegungen des Bodens hinter der sich drehenden Wand kinematisch nur durch plastisch deformierte Scherzonen möglich ist (Bild 2) und nicht mit starren Gleitkörpern und dünnen Scherfugen dargestellt werden kann. Die Deformation der Scherzonen beruht auf der Lösung von Spencer [16]. Bildlich kann man sich z.B. für die Scherzone II in Bild 2 vorstellen, dass jede Raute des dort dargestellten Charakteristikennetzes als Einfachscherung deformiert wird (Details in [4]). Damit ist eine Rotation der Wand möglich. Die Randgleitlinie (BH in Bild 2. die tiefe Gleitfuge) zwischen Fußpunkt und Anker ist hier eine Gerade. Allerdings weist Goldscheider darauf hin, dass infolge von Feinheiten der Randbedingungen physikalischer Experimente (Verschieblichkeit des Wandfußes, Auslösen des Versagens) und durch Effekte des progres- siven Versagens die sichtbare Randgleitlinie auch leicht gekrümmt sein kann. Er postuliert, dass diese Krümmung auf die Geländebruchsicherheit kaum einen Einfluss hat. Berechnungen mit logarithmischen Spiralen als Randgleitlinie können laut Jelinek u. Ostermayer [7] leicht geringere Sicherheiten ergeben als Berechnungen mit geraden Randgleitlinien.

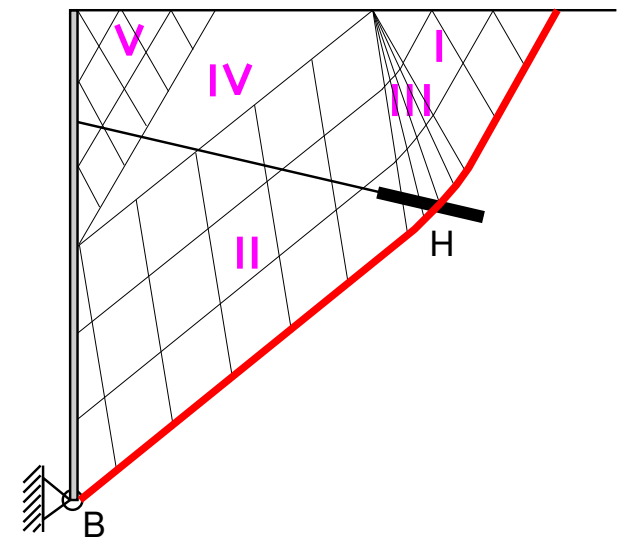

Bild 2: Zusammengesetzter Scherzonenmechanismus aus plastisch deformierenden flächige Scherzonen (I, II, III und V) und einem Starrkörper (IV) hinter der drehenden Wand, nach [4] mit Änderungen.

Weiters zeigt Goldscheider [4], dass die Statik des zusammengesetzten Scherzonenmechanismus (Bild 2) formal mit demjenigen eines Starrkörperbruchmechanismus (Bild 3) übereinstimmt, und damit statisch äquivalent ist. In Bild 3 finden wir die Randgleitlinie (rot) aus Bild 2 wieder. Am Erdwiderlager wirkt der zu einem gewissen Grad mobilisierte passive Erddruck. Die Anwendung der Starrkörperbruchmechanismen zur Berechnung verankerter Stützwände wurde bereits von Goldscheider u. Kolymbas [5] vorgestellt.

In Experimenten mit liegender Ankerplatte (Bild 1 [18, 19]) sowie in numerischen Berechnungen bei ebenem Verzerrungszustand (in denen ein Anker ja einer liegenden Ankerplatte entspricht, z.B. [10, 11]) verläuft die vom Fußpunkt der Wand ausgehende Gleitfuge bis zum Ende der Ankerplatte. In räumlichen Problemen wird allerdings von einer zwischen den Ankern zur Wand hin gekrümmten Gleitfuge ausgegangen, Bild 4. Zusätzlich reicht die tiefe Gleitfuge direkt am Anker in Experimenten mit Einzelankern nie bis ganz ans Ende des Verpresskörpers, siehe Diskussion 


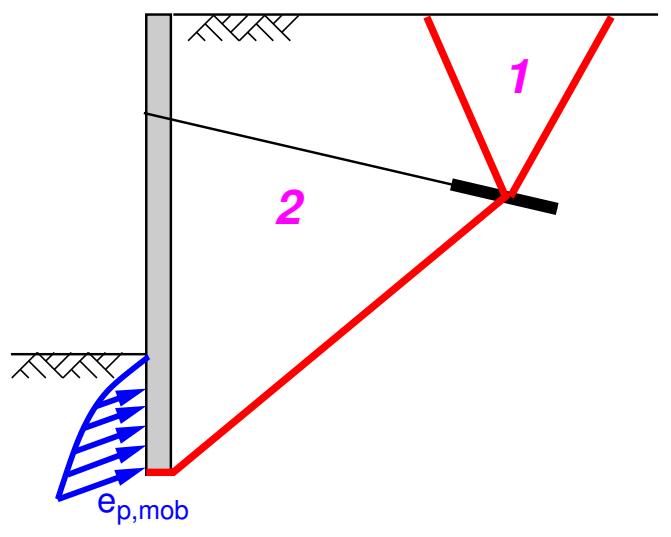

Bild 3: Statisch zu Bild 2 äquivalentes System aus starren Bruchkörpern $(1,2)$ hinter der Wand, nach [4] mit Änderungen.

in [6]. Im statischen Nachweis wird die doppelt gekrümmte tiefe Gleitfuge näherungsweise durch eine Ebene ersetzt und pragmatisch bis zur Mitte des Verpresskörpers angesetzt [2, 6]. Ansätze für andere Verankerungstypen sind in der EAB (2012) angeführt.

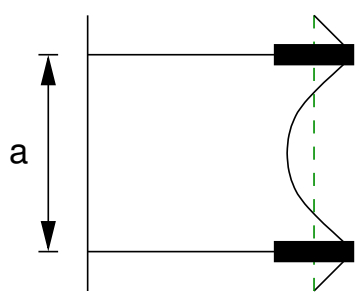

Bild 4: Zur Wand hin gekrümmte tiefe Gleitfuge in der Draufsicht bei Ankern mit großem horizontalem Abstand $a$ und Ersatzgleitfuge (strichliert) in der Mitte der Verpressstrecke.

\subsection{Statik}

In der folgenden Betrachtung wird zur Vereinfachung von einem unbelasteten ebenen Gelände, einer gewichtslosen Wand, die keine Sohlreaktion auslöst, und einem kohäsionslosen Boden ausgegangen. Dies ist allgemein genug, um die einzuführenden Sicherheiten zu diskutieren. Allgemeinere Fälle werden von Goldscheider [4] ausführlich dargestellt.

Für die statische Untersuchung benutzen wir den Starrkörperbruchmechanismus nach Bild 3 . der ja wie bereits erwähnt statisch äquiva- lent zum zusammengesetzten Scherzonenmechanismus (Bild 2) ist. Dabei schneiden wir zunächst zwischen Wand und Erdwiderlager (äußerer Schnitt) und setzen dort die aus der Statik bekannte Lagerkraft $B$ an, die gleich dem mobilisierten passivem Erddruck $E_{\mathrm{p} \text {,mob }}$ ist, welcher wegen der eingeführten Sicherheiten beim Nachweis des Erdwiderlagers kleiner als der maximal mobilisierbare passive Erddruck ist. Der Starrkörper (1) in Bild 3 entspricht in der Geometrie der Scherzone I in Bild2, und kann damit durch einen senkrechten Schnitt mit einem horizontal wirkenden Erddruck nach Rankine ersetzt werden. Im Übrigen ist der durch einen senkrechten Schnitt begrenzte Erdkörper mit horizontalem aktivem Erddruck (Erddruck nach Rankine) statisch äquivalent zu den beiden Starrkörpern (1) und (2) [4. Zuletzt schneiden wir den Erdbereich unterhalb der tiefen Gleitfuge weg und legen damit eine Reaktionskraft $Q$ frei, welche im Grenzzustand die Resultierende aus der maximal mobilisierbaren Reibungskraft $R_{\varphi}=N \tan \varphi$ in der tiefen Gleitfuge und der hierfür maßgebenden Normalkraft $N$ ist. $Q$ ist deshalb um $\varphi$ zur Flächennormalen entgegen der Bewegungsrichtung geneigt. Der so freigelegte Erdkörper und die wirkenden Kräfte sind in Bild 5 dargestellt. Obwohl hier ein Teil des Ankers noch im ruhenden Boden eingezeichnet ist, wird keine Restankerkraft angesetzt, da die Lage der tiefen Gleitfuge zur Berücksichtigung der räumliche Tragwirkung der Einzelanker eben so gewählt wurde, vgl. vorhergehender Abschnitt.

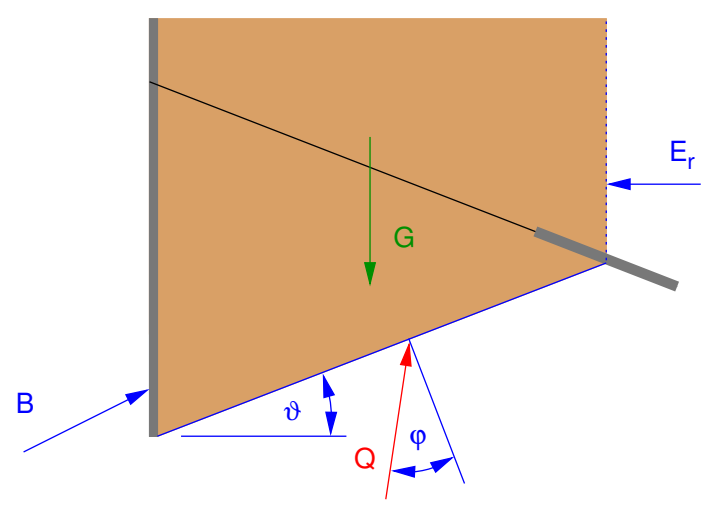

Bild 5: Äußerer Schnitt: Erdköper und Kräfte.

Nun können die bekannten Kräfte, das Gewicht des Erdkörpers $G$, die dem mobilisierten passiven 
Erddruck entsprechende Lagerkraft $B$ und der aktive Erddruck nach Rankine $E_{\mathrm{r}}$, in ein Krafteck gezeichnet werden. Von der Kraft $Q$ ist die Richtung der Wirkungslinie bekannt. Die Wirkungslinie kann ebenfalls eingezeichnet werden, Bild 6 .

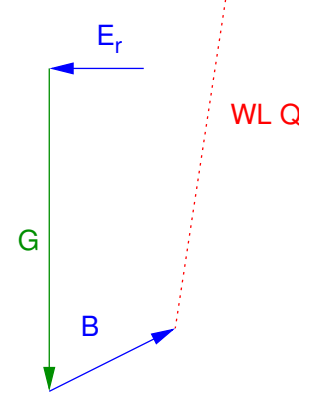

Bild 6: Krafteck zum äußeren Schnitt (Bild 5) mit allen bekannten Elementen.

In dem dargestellten Fall schließt sich das Krafteck nicht. Es kann noch eine zusätzliche Zugkraft am Erdkörper wirken, um einen Grenzzustand herbeizuführen. Eine andere Möglichkeit ist, das Krafteck zunächst mit einer Kraft $Q$ zu schließen, die allerdings nur um den mobilisierte Reibungswinkel $\varphi_{\text {mob }}<\varphi$ geneigt ist. Durch eine zusätzlich Zuggraft wird dieser mobilisierte Reibungswinkel dann bis zum maximal möglichen erhöht. Wir wollen diese Zugkraft hier „zufällig““ in Richtung des Ankers annehmen. Es folgt damit eine zum Versagen notwendige Zugkraft $\Delta Z$, Bild 7.

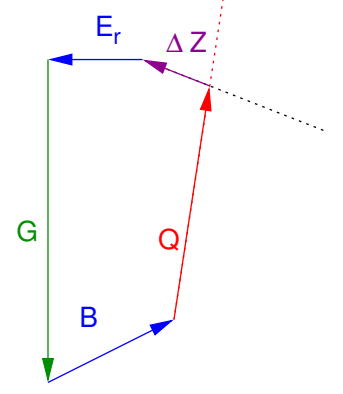

Bild 7: Krafteck zum äußeren Schnitt (Bild 5) im Grenzzustand mit zusätzlicher Zugkraft $\Delta Z$ in Richtung des Ankers.

Wenn, wie in den EAB (2012) [2], zwischen Wand und Erdkörper geschnitten wird (innerer Schnitt), werden die Ankerkraft $A$ und der aktive Erddruck $E_{\mathrm{a}}$ auf die Wand freigelegt, Bild 8 .
Die Lagerkraft $B$ ist im Gleichgewicht mit diesen beiden Kräften, d.h. es gilt die Vektorsumme $B=E_{\mathrm{a}}+A$, Bild 9. Die zum Versagen notwendige Zugkraft $\Delta Z$ bleibt gleich. Dies gilt auch allgemein [4], denn bei Berücksichtigung der Gewichtskraft der Wand $G_{\mathrm{w}}$ und einer Sohlreaktion $Q_{\mathrm{w}}$, steht $B$ mit diesem auch im Gleichgewicht: $B=E_{\mathrm{a}}+A+G_{\mathrm{w}}+Q_{\mathrm{w}}$. Es ist also statisch egal, auf welcher Seite der Wand geschnitten wird. Dies wurde bereits von Schulz [14] angemerkt. Da die Kraft $B$ allerdings als Reaktionskraft aus der Statik des Verbaus berechnet werden muss, ist es rechentechnisch einfacher, den inneren Schnitt zu führen.

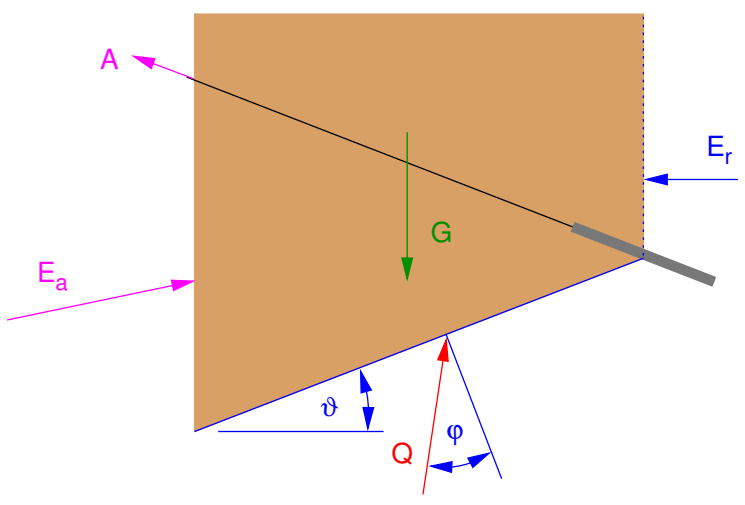

Bild 8: Innerer Schnitt: Erdkörper und Kräfte.

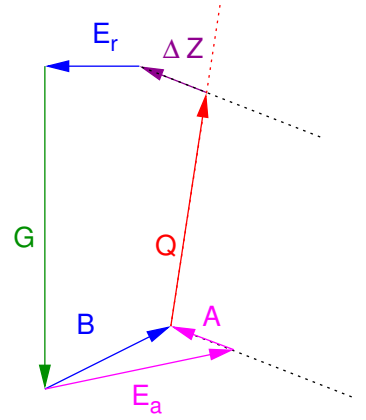

Bild 9: Krafteck zum inneren Schnitt (Bild 80 im Grenzzustand mit zusätzlicher Zugkraft $\Delta Z$ in Richtung des Ankers.

In den EAB (2012) [2] wird das Krafteck ohne die Ankerkraft $A$ gezeichnet (Verfahren nach Kranz [9]) und das Krafteck mit einer Kraft in Ankerrichtung geschlossen, welche als mögliche Ankerkraft $A_{\text {mögl }}$ bezeichnet wird, Bild 10. Diese Kraft ist offensichtlich

$$
A_{\text {mögl }}=A+\Delta Z \text {. }
$$




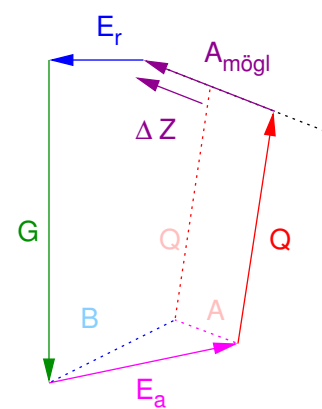

Bild 10: Krafteck zum inneren Schnitt (Bild 8) nach Kranz, bzw. EAB (2012). Mit punktierten Linien sind die Kräfte aus Bild 9 hinzugefügt.

\section{Sicherheit}

Das Bruchmodell in Bild 5 oder Bild 8 kann als Standsicherheitsproblem für einen trapezförmigen Bodenkörper angesehen werden [20, S. 289], und sollte deshalb eigentlich im Nachweisverfahren 3 (Gesamtstandsicherheit) nach Eurocode 7 behandelt werden, [vgl. 21, S. 288]. Dazu müssten die Teilsicherheiten auf die Scherparameter des Bodens angewandt werden, und dann geprüft werden, ob sich das Krafteck (Bild 7 oder 9 ) mit den so neu zu berechnenden Größen der Erddrücke und der Ankerkraft unter Einführung einer zusätzlichen destabilisierend wirkenden Zugkraft $\Delta Z \geq 0$ schließen lässt. Weil dazu die Berechnung der Erddrücke und der Statik des Baugrubenverbaus wiederholt werden muss, wird vereinfacht in den derzeit gültigen Regelwerken (EAB [2] und EAU [3]) das Nachweisverfahren 2 (GEO-2) angewandt.

\subsection{Nachweis der tiefen Gleitfuge}

Für den Nachweis im Verfahren 2 laut Eurocode 7 wird mit charakteristischen Werten gerechnet und laut EAB (2012) mit dem Krafteck in Bild 10 eine mögliche charakteristische Ankerkraft $A_{\text {mögl lk }}$ bestimmt. Aus der Statik der Wand ist die charakteristische Ankerkraft $A_{\mathrm{k}}$ bekannt. Der Nachweis wird mit Bemessungswerten geführt

$$
A_{\mathrm{d}} \leq A_{\mathrm{mög} ; \mathrm{d}},
$$

welche mittels Teilsicherheiten aus den charakteristischen Werten berechnet werden [z.B. 15,
C 9.2.9], wobei der Bemessungswert der möglichen Ankerkraft mit dem Teilsicherheitsbeiwert für den passiven Erddruck $\gamma_{\mathrm{R}, \mathrm{h}}$ berechnet wird [15], um keine weiteren Zahlenwerte einführen zu müssen. Klar ist, dass mit diesem Vorgehen die vorhandene Ankerkraft als Beanspruchung und die mögliche Ankerkraft als Widerstand betrachtet wird.

Der Nachweis (2) ist laut (1) gleichbedeutend mit $\Delta Z_{\mathrm{d}} \geq 0$, einer zusätzlichen destabilisierend wirkenden Zugkraft in Ankerrichtung. In diesem Sinne ist der Nachweis also prinzipiell so möglich, und wird in der Praxis für den Nachweis von Baugrubenwänden verwendet, siehe u.a. [2, 1].

Es wird üblicherweise ein Ausnutzungsgrad des Ankers definiert [20, 15]

$$
\nu=\frac{A_{\mathrm{d}}}{A_{\mathrm{möglj} ; \mathrm{d}}} .
$$

Spätestens hier wird klar, dass das Vorgehen dem ursprünglichen Ansatz einer globalen Sicherheit von Kranz [9] entspricht

$$
\eta=\frac{A_{\text {mögl }}}{A},
$$

welche für schlaffe Anker und eine dreicksförmige Erddruckverteilung eingeführt wurde.

Für ständige Lasten alleine folgt direkt

$$
\nu=\frac{A_{\mathrm{d}}}{A_{\text {mögl; }}}=\frac{\gamma_{\mathrm{G}} A}{A_{\text {mögl }} / \gamma_{\mathrm{R}, \mathrm{h}}}=\frac{\gamma_{\mathrm{G}} \gamma_{\mathrm{R}, \mathrm{h}}}{\eta},
$$

also im rechnerischen Grenzzustand $\nu=1$

$$
\eta=\gamma_{\mathrm{G}} \gamma_{\mathrm{R}, \mathrm{h}} .
$$

Damit ist das bisher gewohnte Sicherheitsniveau (zumindest formal) leicht im neuen Sicherheitskonzept zu erhalten. Streng genommen wird mittels (4) eine mögliche externe Zuggkraft der GröBe $\Delta Z=A(1+\eta)$ als gerade noch zulässig angesehen. Die Sicherheitsdefinition in (4) wurde bereits von Jelinek u. Ostermayer [7] und Schulz [14] kritisiert. Es dürfte zumindest mit einem äußeren Schnitt (Bild 5), der ja statisch äquivalent zum inneren Schnitt (Bild 8) ist, sofort klar sein, dass die vorhanden Ankerkraft als innere Kraft keinen Einfluss auf die Standsicherheit des in der Modellierung verwendeten starren Bruchkörpers hat, 
vgl. auch [8, S. 445]. Um diesen offensichtlichen Umstand zu umgehen, wird argumentiert: „Die Sicherheitsdefinition über die Ankerkraft hat daher eine Stellvertreterfunktion für die Ausnutzung der Scherfestigkeit des Bodens." [13, S. 320], vgl. sinngemäß auch [3, S. 491]. Die so definierte Ausnutzung ist aber nicht direkt mit der Ausnutzung des Widerstandes in der Scherfuge vergleichbar, vgl. dazu ein analoges Beispiel in Anhang A.

\subsection{Problempunkte beim Nachweis nach Kranz}

Es können folgende Problempunkte genannt werden, welche aus der Modellbildung nach Kranz folgen:

1. Die Sicherheitsdefinition über die Ankerkraft, die als innere Kraft im betrachteten Starrkörper keinen Einfluss auf dessen Standsicherheit haben kann.

2. Die (wenn auch als indirekt deklarierte) Verwendung einer möglichen Ankerkraft als Widerstand im Nachweis, anstelle direkt - und damit weitaus verständlicher - den mechanisch offensichtlich wirkenden Widerstand in der tiefen Gleitfuge zu verwenden.

3. Durch die Definition einer möglichen Ankerkraft kann das falsche Bild entstehen, dass die Ankerkraft durch das Versagen des Bodenkörpers in der tiefen Gleitfuge begrenzt ist. Die Ankerkraft wird hingegen mechanisch durch den Ausziehwiderstand des Verpresskörpers begrenzt.

Alle diese Komplikationen können leicht durch eine andere Modellbildung ausgeräumt werden.

\section{Alternative Nachweise der tiefen Gleitfuge}

\subsection{Reduktion der Scherparameter (GEO-3)}

Am verständlichsten wäre es natürlich, den Nachweis der tiefen Gleitfuge nach dem Nachweisverfahren $3 \mathrm{zu}$ führen (Verlust der Gesamtstandsicherheit, Reduktion der Scherparameter). Der
Bruchkörper ist im Nachweis durch die Abmessungen der Wand und des Ankers gegeben. Die Form ist also unabhängig von den Bodeneigenschaften, nicht wie zum Beispiel die Form eines aktiven Erddruckkeiles. Damit fällt eines der üblichen Argumente gegen dieses Verfahren weg. In Zeiten der computergestützten Berechnung sollte auch das Argument des Mehraufwandes durch zwei getrennte Rechnungen nicht zu schwer wiegen.

Dieser Nachweis wird auch für die Bemessung der Nagelwand verwendet und im Folgenden als Referenz zur Beurteilung des Sicherheitsniveaus verwendet.

\subsection{Beanspruchung und Widerstand in der Scherfuge}

Da derzeit allerdings das Nachweisverfahren 2 umgesetzt werden muss, sollte dies durch den Vergleich der Beanspruchung in der Scherfuge mit dem Widerstand in der Scherfuge erfolgen. Dies ist auch in völliger Übereinstimmung mit den Nachweiskonzepten des Eurocodes 7, und wurde für den Fall einer auf erhöhten aktiven Erddruck bemessenen Wand in Anhang H der E DIN 1054 (12/2000) beschrieben [17]. Der direkte Vergleich von Beanspruchung und Widerstand in einer maßgebenden Scherfuge (hier die tiefe Gleitfuge) lässt sich im Übrigen auch wesentlich leichter vermitteln, als ein indirekter Nachweis über eine Ankerkraft. Damit wird das Verständnis der allgemeinen Modellierung von Versagenszuständen in der Geotechnik geschult.

Graphisch erhält man die Beanspruchung in der Gleitfuge aus dem Krafteck in Bild 11. Die Summe der Einwirkungen $G, E_{\mathrm{r}}, E_{\mathrm{a}}$ und $A$ wird in eine Komponente $E$ parallel zur tiefen Gleitfuge und eine Komponente $N$ normal dazu zerlegt. $E$ ist die Beanspruchung, und aus $R_{\varphi}=N \tan \varphi$ folgt der Anteil der Reibung am Widerstand. Eine Kohäsionskraft kann einfach addiert werden, da sie in die gleiche Richtung wirkt. Der Nachweis ist auch rechnerisch einfach (vgl. Beispiel in [17]).

Es gibt nun zwei Möglichkeiten, die Teilsicherheiten anzuwenden. Diese können wie derzeit in der DIN eingeführt direkt die Beanspruchung (de- 


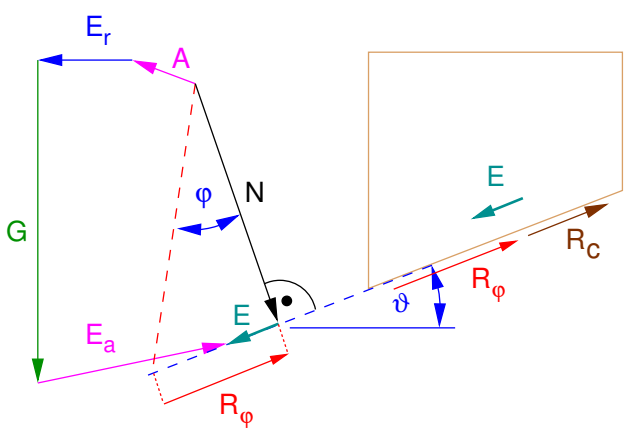

Bild 11: Krafteck zum inneren Schnitt (Bild 8). Ermittlung der Einwirkung $E$ und der Normalkomponente $N$ aus den Einwirkungen sowie die am Bruchkörper in der Gleitfuge wirkende Kräften: $E, R_{\varphi}=$ $N \tan \varphi$ und ein allfälliger Widerstand aus Kohäsion $R_{c}$.

sign aproach $2^{*}$, DA $2^{*}$ ) erhöhen oder, wie allgemein im Eurocode auch möglich, die Einwirkungen (DA 2).

\subsubsection{Design approach 2* (DA 2*)}

Von den am Bruchkörper angreifenden charakteristischen Einwirkungen werden die Vertikalkomponenten und die Horizontalkomponenten summiert: $\sum V_{\mathrm{k}}$ und $\sum H_{\mathrm{k}}$. Dabei kann zwischen ständigen und veränderlichen Einwirkungen getrennt werden, da hierfür verschiedene Teilsicherheitsbeiwerte angewendet werden müssen. Die charakteristische Beanspruchung aus den summierten Einwirkungen in der um $\vartheta$ geneigten tiefen Gleitfuge ist

$$
E_{\mathrm{k}}=\sin \vartheta \cdot \sum V_{\mathrm{k}}-\cos \vartheta \cdot \sum H_{\mathrm{k}},
$$

mit $\sum H_{\mathrm{k}}$ positiv in Richtung $B_{\mathrm{h} ; \mathrm{k}}$, also sozusagen haltend. Die Normalkraft auf die Scherfuge ist

$$
N_{\mathrm{k}}=\cos \vartheta \cdot \sum V_{\mathrm{k}}+\sin \vartheta \cdot \sum H_{\mathrm{k}} .
$$

Der Widerstand in der tiefen Gleitfuge ist

$$
R_{\mathrm{k}}=N_{\mathrm{k}} \tan \varphi+c_{\mathrm{k}} L,
$$

mit der Länge $L$ der tiefen Gleitfuge. Als Teilsicherheitsbeiwert für den Widerstand wird von Walz [17] derjenige für den Gleitwiderstand verwendet. Der Nachweis erfolgt dann formal

$$
E_{\mathrm{d}} \leq R_{\mathrm{d}} .
$$

Der Ausnutzungsgrad ist wie üblich $\nu=E_{\mathrm{d}} / R_{\mathrm{d}}$.

\subsubsection{Design approach 2 (DA 2)}

Hier werden die charakteristischen Einwirkungen in Bemessungswerte umgewandelt und deren Vertikalkomponenten und die Horizontalkomponenten summiert: $\sum V_{\mathrm{d}}$ und $\sum H_{\mathrm{d}}$. Damit folgt direkt der Bemessungswert der Beanspruchung

$$
E_{\mathrm{d}}=\sin \vartheta \cdot \sum V_{\mathrm{d}}-\cos \vartheta \cdot \sum H_{\mathrm{d}} .
$$

Die zugehörige Normalkraft ist

$$
N_{\mathrm{d}}=\cos \vartheta \cdot \sum V_{\mathrm{d}}+\sin \vartheta \cdot \sum H_{\mathrm{d}} .
$$

Der Widerstand in der tiefen Gleitfuge ist

$$
R_{\mathrm{d}}=N_{\mathrm{d}} \tan \varphi+c_{\mathrm{k}} L / \gamma_{\mathrm{R}, \mathrm{h}} .
$$

\subsubsection{Erweiterung auf mehrere Ankerlagen}

Eine logische Erweiterung auf mehrere Ankerlagen kann mit dem auf Ranke u. Ostermayer [12] basierendem Prozedere der EAB [2] erfolgen. Dazu werden die in den EAB empfohlene Schnitte durchgeführt, d.h. jeder Mittelpunkt einer Krafteinleitungsstrecke ist einmal als Endpunkt einer tiefen Gleitfuge anzunehmen.

Die Kräfte von geschnittenen Ankern werden voll $A$ bzw. nur anteilig $A^{\star}$ angesetzt, je nachdem ob der Schnitt in der freien Länge oder der Krafteinleitungsstrecke $l_{0}$ erfolgt. Die anteilige Kraft bei Schnitt in einer Krafteinleitungsstrecke ergibt sich aus einer Annahme einer gleichmäßigen Verteilung der Mantelreibung entlang der Krafteinleitungsstrecke. Mit der Länge der Krafteinleitungsstrecke im ruhenden Boden $l_{0}^{\star}$ : $A^{\star}=A l_{0}^{\star} / l_{0}[4]$.

Als Schnitte sind die tiefe Gleitfuge, die Gleitfuge des aktiven Gleitkeils, der die Erddruckkraft auf die Ersatzankerwand hervorruft, und gegebenenfalls der Schnitt hinter der Stützwand beim einem inneren Schnitt zu betrachten. Das bedeutend bei einem inneren Schnitt, dass sich Ankerkräfte von zwei mal in der freien Länge geschnittenen Ankern aufheben. Diese Anker müssen nicht weiter berücksichtigt werden, genau so wie im Nachweis nach EAB. Von den Kräften der Anker, deren zweiter Schnitt in der Krafteinleitungsstrecke liegt, verbleibt $P=A-A^{\star}$, also wie in der EAB der Anteil der Ankerkraft im Gleitkörper. Anker, deren Verpresskörper ganz im Gleitkörper 
liegen, werden nur einmal geschnitten und damit voll angesetzt $P=A$. Damit ergeben sich in Summe für die weiteren Anker dieselben Kräfte $P$ wie in $\operatorname{EAB}$ (2012) 7.3 10.b) und c).

Die verbleibenden Ankerkräfte $P$ sind in der Bildung der Summen für die Vertikal- und Horizontalkräfte zu berücksichtigen. Die Berechnung der Beanspruchung und des Widerstandes in der tiefen Gleitfuge aus $\sum V$ und $\sum H$ bleibt dann formal gleich.

\section{Vergleichsrechnungen}

Zur Veranschaulichung der Auswirkung verschiedener Ansätze zur Berücksichtigung der Sicherheiten wird das Beispiel im Kommentar zum Handbuch Eurocode 7 [15, C 9.2.9] verwendet, Bild 12 .

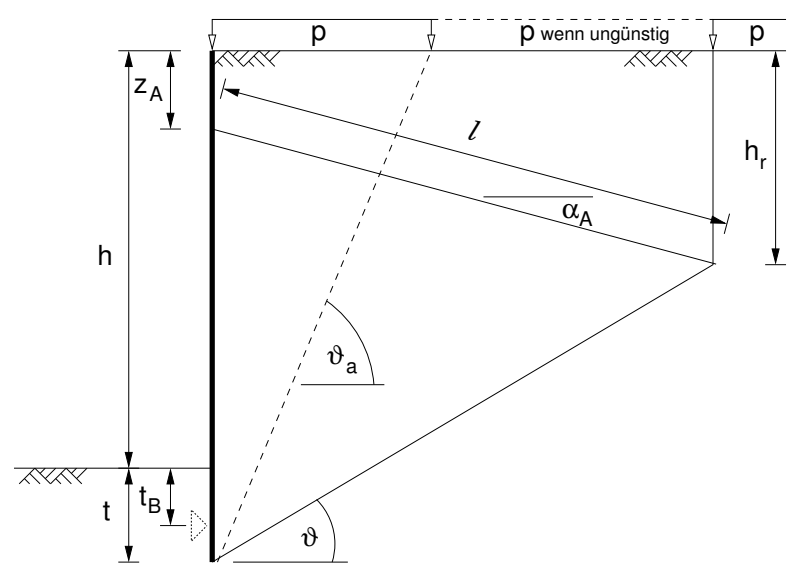

Bild 12: Beispiel.

Der Baugrubenverbau hat eine freie Höhe $h=$ 8, $0 \mathrm{~m}$ und eine rechnerische Einbindetiefe für freie Lagerung im Boden $t=1,8 \mathrm{~m}$. Der Angriffspunkt der Auflagerkraft $B$ liegt bei $t_{B}=1,1 \mathrm{~m}$, der Anker liegt bei $z_{A}=1,5 \mathrm{~m}$ und ist um $\alpha=15^{\circ}$ geneigt. Die Länge des Ankers bis zum Schwerpunkt des Verpresskörpers ist $l$. Der Boden hat eine Wichte von $\gamma=17 \mathrm{kN} / \mathrm{m}^{3}$ und die Scherparameter $\varphi=35^{\circ}$ und $c=0$. Die Neigung des aktiven Erddruckes ist $\delta_{a}=2 / 3 \varphi$. Der aktive Erddruck oberhalb der Baugrubensohle wird nach EAB umgelagert. Die Auflast $p=10 \mathrm{kN} / \mathrm{m}^{2}$ wird beim Nachweis GEO-2 (Kranz) im Bereich zwischen der Gleitfläche des Erdkeiles $\left(\vartheta_{\mathrm{a}}\right)$ des aktiven Erddruckes und der Ersatzankerwand nur angesetzt, wenn $\vartheta>\varphi$ ist, da diese Auflast ansonsten günstig wirkt. Für die anderen Nachweise wirkt dieser Teil der Auflast ungünstig, wenn die Summe der Horizontalkräfte stützend für den Bruchkörper wirkt, vgl. Bild 20 in Anhang A.

Für alle im Folgenden durchgeführten Berechnungen mit geänderten Parametern ergibt sich mit der Einbindetiefe $t=1,8 \mathrm{~m}$ eine Ausnutzung des Erdauflagers $\nu_{B} \leq 1$. Auf eine Optimierung der Einbindetiefe wurde aus Vergleichbarkeitsgründen verzichtet.

Zunächst wird eine Parmaterstudie für die temporäre Bemessungssituation BS-T durchgeführt, denn dafür ist nach (6) $\gamma_{\mathrm{G}} \gamma_{\mathrm{R}, \mathrm{e}}=1,20 \cdot 1,30=$ $1,56 \approx 1,5=\eta_{\mathrm{Kranz}}$, also die Sicherheit für den Nachweis der tiefen Gleitfuge im alten, globalen Sicherheitskonzept. Zum Vergleich der Verfahren wurden jeweils jene minimal erforderlichen Ankerlängen berechnet, für welche die jeweiligen Nachweise der tiefen Gleitfuge gerade den Ausnutzungsgrad $\nu=1$ ergeben. Der Nachweis für ständige Lasten alleine ergibt die erforderlichen Ankerlängen in Bild 13. Dabei zeigt sich, dass das Verfahren der E DIN 1054:2000 zu deutlich längeren Ankern führen würde, als die drei anderen, die sehr ähnlich Ankerlängen ergeben.
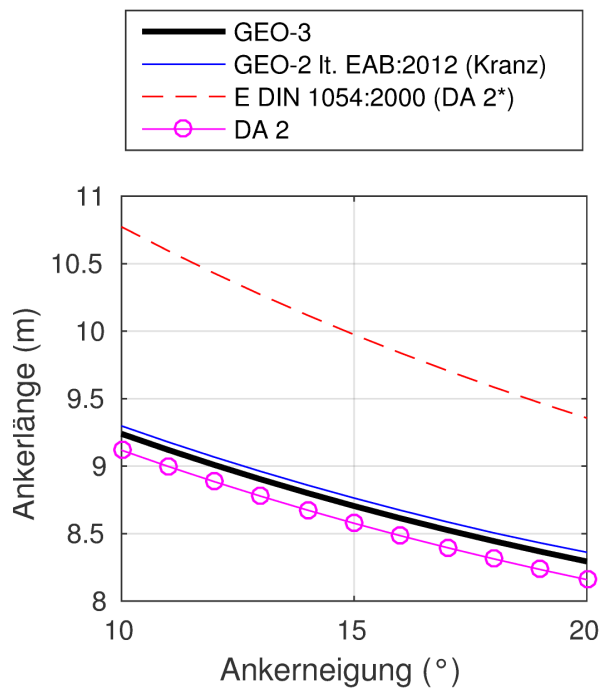

Bild 13: Länge des Ankers $l$ als Funktion der Ankerneigung für $\nu=1$, BS-T, freie Wandhöhe $h=8,0 \mathrm{~m}$, Anker $z_{A}=1,5 \mathrm{~m}$ unter der Geländeoberfläche, Erddruck nach EAB umgelagert.

Als Vergleich zwischen den Verfahren wird nun 
die Differenz der jeweiligen minimalen Ankerlänge zur minimalen Ankerlänge aus dem Verfahren GEO-3 in Prozent berechnet. Positive Werte bedeuten vergleichsweise längere Anker, negative Werte bedeuten kürzere Anker. Für alle Kombinationen der Eingangswerte $p=0$ bis $p=10 \mathrm{kN} / \mathrm{m}^{2}, \alpha=0$ bis $25^{\circ}, z_{A}=0,1 h$ bis $0,2 h$ und $\varphi=33^{\circ}$ bis $40^{\circ}$ ergibt dies für das Verfahren GEO-2 (Kranz) prozentuale Differenzen von $-1.0 \%$ bis 2,5\% zu den Ankerlängen aus dem Verfahren GEO-3, für das Verfahren nach DA $2^{*}$ der E DIN 1045:2012 folgen Differenzen von $10.0 \%$ bis $25.8 \%$ und für den Nachweis nach DA 2 ergeben sich Differenzen von $-1.9 \%$ bis $0.6 \%$.

Etwas anders fallen die Vergleiche für die permanente Bemessungssituation (BS-P) und die außergewöhnliche Bemessungssituation (BS-A) aus. Die Ankerlängen für den Nachweis nach DA 2 ändern sich nicht, siehe Tabelle 1 und die Differenzen zu den Ankerlängen aus GEO-3 ist deutlich größer als im BS-T. Dies liegt vor allem daran, dass der Teilsicherheitsbeiwert $\gamma_{\mathrm{R}, \mathrm{h}}=1,10$ für alle Bemessungssituationen gleich ist.

Durch eine leichte Anpassung der Sicherheitsbeiwerte auf $\gamma_{\mathrm{R}, \mathrm{h}}=1,25$ für BS-P und $\gamma_{\mathrm{R}, \mathrm{h}}=$ 1, 15 für BS-T (also auf die Werte für $\gamma_{\varphi}$ ) verschieben die Differenzen für das verfahren DA 2 durchwegs in den positiven (konservativen) Bereich (Tabelle 2). Das Verfahren nach Kranz kann im Fall kohäsiver Böden allerdings auch kürzere Anker als das Verfahren GEO-3 liefern, siehe Bild 14 für das hier betrachtet Beispiel mit $c=10 \mathrm{kN} / \mathrm{m}^{2}$ im BS-P maximal um $3,2 \%$ kürzere Anker. Für $c=20 \mathrm{kN} / \mathrm{m}^{2}$ und $\varphi=25^{\circ}$ ergibt sich ein um 6,1\% kürzerer Anker.

\begin{tabular}{|l|c|c|c|}
\hline & BS-P & BS-T & BS-A \\
\hline GEO-3 & 9,23 & 8,70 & 8,43 \\
GEO-2 (Kranz) & 9,27 & 8,76 & 8,40 \\
& $(+0,4 \%)$ & $(+0,7 \%)$ & $(-0,4 \%)$ \\
DA 2 & 8,58 & 8,58 & 8,58 \\
& $(-7.1 \%)$ & $(-1,5 \%)$ & $(+1,7 \%)$ \\
DA 2* (E DIN) & 10,82 & 9,88 & 9,21 \\
& $(+17 \%)$ & $(+14 \%)$ & $(+9,2 \%)$ \\
\hline
\end{tabular}

Tabelle 1: Ankerlängen $l$ in Meter für $\nu=1$, in Klammer: prozentuale Abweichungen zu den Längen für GEO-3.

\begin{tabular}{|l|c|c|c|}
\hline & BS-P & BS-T & BS-A \\
\hline GEO-3 & 9,23 & 8,70 & 8,43 \\
DA 2 & 9,46 & 8,91 & 8,58 \\
& $(+2,4 \%)$ & $(+2,3 \%)$ & $(+1,7 \%)$ \\
\hline
\end{tabular}

Tabelle 2: Ankerlängen $l$ in Meter für $\nu=1$ mit $\gamma_{\mathrm{R}, \mathrm{h}}=1,25=\gamma_{\varphi}$ für BS-P und $\gamma_{\mathrm{R}, \mathrm{h}}=1,15=\gamma_{\varphi}$ für BS-T, in Klammer: prozentuale Abweichungen zu den Längen für GEO-3.
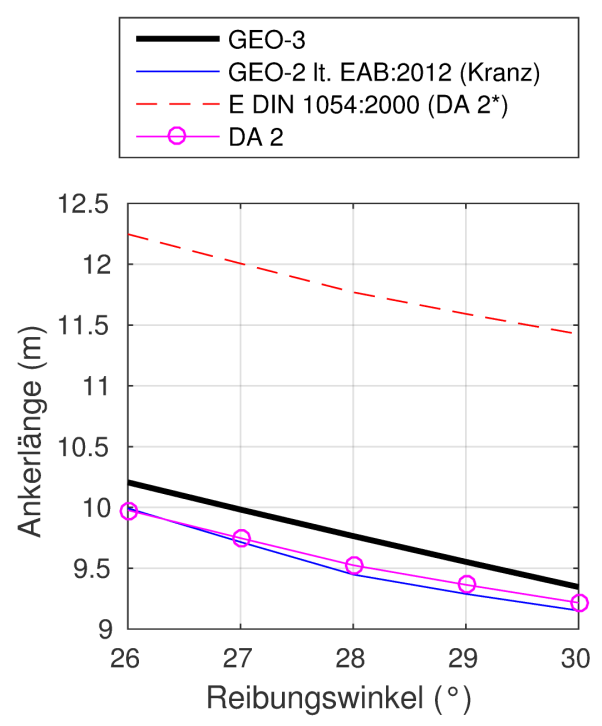

Bild 14: Länge des Ankers $l$ als Funktion des Reibungswinkels mit $c=10 \mathrm{kN} / \mathrm{m}^{2}$ für $\nu=1$, BS-P. Die Knicke in den Verläufen entstehen durch den Wechsel auf den minimalen Erddruck. 


\section{Zusammenfassung}

Die Geometrie des Versagensmodells zum Nachweis der tiefen Gleitfuge ist gut begründet. Lediglich die Einführung der Sicherheiten sollte überdacht werden. Die derzeit wieder gewählte Version über eine sogenannte mögliche Ankerkraft ist mechanisch nicht konform mit den sonstigen Nachweisen im Nachweisverfahren 2 des Eurocodes, da der beanspruchte Bereich offensichtlich in der tiefen Gleitfuge liegt und demzufolge auch dort der Nachweis geführt werden muss. Ein solcher Nachweis wurde bereits vorgeschlagen, hat aber keinen Eingang in die aktuellen Regelwerke gefunden. Der indirekte Nachweis der Beanspruchung der tiefen Gleitfuge über die Ankerkraft ist nur oberflächlich einfach, aber im Detail problematisch.

An einem Beispiel mit zum Teil variierten Eingangsparametern wurde gezeigt, dass die Ankerlängen aus einer Bemessung mit dem derzeitigen auf der Sicherheitsdefinition von Kranz beruhendem GEO-2 Verfahren und ein Nachweis nach design approach 2 (DA 2) nur um wenige Prozent zu Ankerlängen differieren, welche sich aus einer Bemessung nach dem Verfahren GEO-3 ergeben würden. Das in E DIN 1054:2000 vorgeschlagen Verfahren nach design approach $2 *$ ergibt wesentlich längere Anker.

Ein Wechsel auf einen Nachweis nach GEO-3 wäre wünschenswert, da es sich offensichtlich um ein Problem der Gesamtstandsicherheit handelt. Damit wäre das Sicherheitsniveu vergleichbar mit allen anderen Nachweisen dieser Klasse. Solange das nicht geht, bestünde die Möglichkeit das derzeitige GEO-2 (Kranz) Verfahren durch ein Verfahren DA $2 \mathrm{zu}$ ersetzten. Mit den derzeitigen Teilsicherheitsbeiwerten für den Gleitwiderstand ergeben sich im betrachteten Beispiel für kohäsive Böden meistens leicht längere Ankerlängen als bisher gewohnt und für kohäsionslose Böden leicht kürzere Ankerlängen. Sollen die gewohnten Ankerlängen möglichst nicht unterschritten werden, müssten die Teilsicherheitsbeiwerte für Gleiten auf diejenigen für den Reibungswinkel angehoben werden.

Danksagung: Ich bedanke mich bei Prof. Dimitrios Kolymbas für anregende Diskussionen und bei den 4 anonymen Gutachtern für hilfreiche Kommentare und Anregungen.

\section{Literatur}

[1] Berhane, G.: Standsicherheitsnachweis in der tiefen Gleitfuge unter Berücksichtigung von Strömungskräften. In: geotechnik 38 (2015), Nr. 3, 205-217. http://dx. doi.org/10.1002/gete.201400037. - DOI 10.1002/gete.201400037. - ISSN 2190-6653

[2] EAB: Empfehlungen des Arbeitskreises „Baugruben“. 5. Deutsche Gesellschaft für Geotechnik e.V., 2012

[3] EAU: Empfehlungen des Arbeitsausschusses,Ufereinfassungen" Häfen und Wasserstraßen. 11. Deutsche Gesellschaft für Geotechnik e.V., 2012

[4] Goldscheider, M.: Zum Nachweis der Geländebruchsicherheit und der erforderlichen Ankerlänge verankerter Stützwände. In: Bautechnik 77 (2000), Nr. 9, S. 641-656

[5] Goldscheider, M. ; Kolymbas, D.: Berechnung der Standsicherheit verankerter Stützwände. In: geotechnik 4 (1980), S. 156164

[6] Heibaum, M. ; Herten, M.: Zuschriften zu Perau, E.: Konzept und FE-Modellierung zum Nachweis der erforderlichen Ankerlängen. In: Bautechnik 85 (2008), Nr. 9, S. 653656

[7] Jelinek, R. ; Ostermayer, H.: Verankerungen von Baugrubenumschließungen. In: Vortragsband zur Baugrundtagung München, 1966, S. 271-310

[8] Kolymbas, D.: Geotechnik : Bodenmechanik, Grundbau und Tunnelbau. 3. Springer, 2011

[9] Kranz, E.: Über die Verankerung von Spundwänden. Ernst \& Sohn, 1953

[10] Perau, E.: Nachweis der erforderlichen Ankerlänge mit der Finite-Elemente-Methode. In: Bautechnik 84 (2007), Nr. 6, S. 367-378 
[11] Perau, E.: Konzept und FE-Modellierung zum Nachweis der erforderlichen Ankerlänge. In: Bautechnik 85 (2008), Nr. 4, S. 247-257

[12] Ranke, A. H. ; Ostermayer, H.: Beitrag zur Stabilitätsuntersuchung mehrfach verankerter Baugrubenumschließungen. In: Die Bautechnik 45 (1968), Nr. 10, S. 341-350

[13] Richwien, W. ; Kalle, H.-U. ; Lambertz, K.-H. ; Morgen, K. ; Vollstedt, H.W.: Spundwände. In: WiTT, K. J. (Hrsg.): Grundbau-Taschenbuch: Teil 3. Ernst \& Sohn, 2010, Kapitel 3.3, S. 279-353

[14] Schulz, H.: Überlegungen zur Führung des Nachweises der Standsicherheit in der tiefen Gleitfuge. In: Mitteilungsblatt der Bundesanstalt für Wasserbau Nr. 41. Bundesanstalt für Wasserbau, 1977, S. 153-171

[15] Schuppener, B. (Hrsg.): Kommentar zum Handbuch Eurocode 7 - Geotechnische Bemessung, Allgemeine Regeln. Ernst \& Sohn, 2012

[16] Spencer, A. J. M.: Deformation of ideal granular materials. In: Mechanics of Solids (1982), S. 607-652

[17] Walz, B.: Nachweis der Standsicherheit in der tiefen Gleitfuge. In: Deutsche Gesellschaft für Geotechnik e. V. DGGT, Arbeitskreis Baugruben: Aktuelle Entwicklungen bei der Berechnung von Baugruben. Dortmund : Deutsche Gesellschaft für Geotechnik e. V. DGGT, 2003, S. 44-53

[18] Walz, B.: Bodenmechanische Anschauungsversuche - Gleitfuge Ankerplatte. Bergische Universität Wuppertal. http: //www.geotechnik.uni-wuppertal.de/ fileadmin/bauing/geotechnik/Filme/ Tiefe_Gleitfuge-Ankerplatte.avi.

Version: 2004

[19] Walz, B.: Möglichkeiten und Grenzen bodenmechanischer 1g-Modellversuche. In: RACKWITZ, Frank (Hrsg.): Entwicklungen in der Bodenmechanik, Bodendynamik und Geotechnik: Festschrift zum 60. Geburtstag von Univ.-Professor Dr.-Ing. habil. Stavros A. Savidis. Berlin, Heidelberg : Springer Berlin Heidelberg, 2006, S. 63-78

[20] Weissenbach, Anton ; Hettler, Achim: Baugruben: Berechnungsverfahren. Ernst \& Sohn, 2010

[21] ZIEgler, M.: Geotechnische Nachweise nach EC 7 und DIN 1054: Einführung in Beispielen. Ernst \& Sohn, 2012 (Bauingenieur-Praxis)

\section{A. Block auf geneigter Ebene}

Das Abrutschen eines Blocks auf einer geneigten Ebene (Bild 15) als Grundbeispiel der Felsmechanik ist mechanisch gleich der Stabilitätsuntersuchung einer unendlichen Böschung, und ist deshalb auch in der Bodenmechanik didaktisch sehr wertvoll. Die Kontaktfläche zwischen Block und Unterlage steht für eine Scherfuge im Boden, wie das auch bei der Methode der Starrkörperbruchmechanismen benutzt wird. Für die folgenden Erläuterungen ist es wesentlich übersichtlicher, globale Sicherheitsfaktoren einzuführen, als mit Teilsicherheiten zu arbeiten.

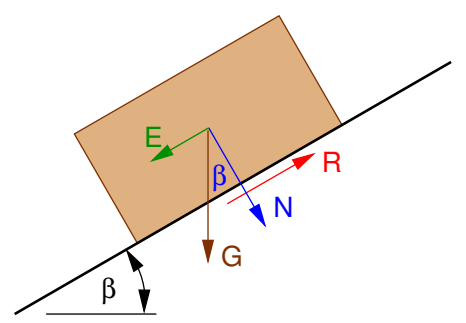

Bild 15: Block auf geneigter Ebene.

Für den Block auf der geneigten Ebene ist unstrittig, dass als Beanspruchung $E=G \sin \beta$ und als Widerstand $R=N \tan \varphi=G \cos \beta \tan \varphi$ definiert wird. Damit ist die Sicherheit

$$
\eta=\frac{R}{E}=\frac{\tan \varphi}{\tan \beta},
$$

welche im Grenzfall $\beta=\varphi$ gleich 1 wird.

Betrachten wir nun den Fall eines Blocks, welcher zusätzlich mit einer Horizontalkraft $H$ belastet wird, Bild 16. Diese kann zum Beispiel 


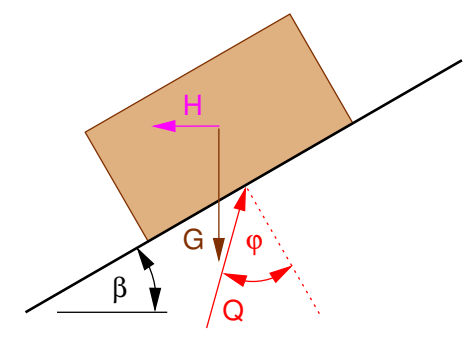

Bild 16: Block auf geneigter Ebene, mit Horizontalkraft $H$ belastet.

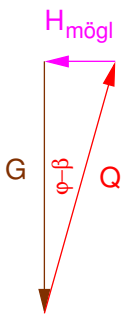

Bild 17: Krafteck zum Block auf geneigter Ebene in Bild 16.

aus einer Erdbebenwirkung stammen. Man kann nun auf die Idee kommen, für diesen Block eine mögliche Horizontalkraft zur Herbeiführung eines Grenzzustandes aus dem Krafteck in Bild $17 \mathrm{zu}$ berechnen

$$
H_{\mathrm{mögl}}=G \tan (\varphi-\beta),
$$

und diese mit der vorhandenen Horizontalkraft zu vergleichen

$$
\eta_{H}=\frac{H_{\mathrm{mögl}}}{H} .
$$

Dies ist eine Sicherheitsdefinition, wie sie im Prinzip von Kranz verwendet wird. Alternativ kann aber auch eine Beanspruchung $E$ aus den Komponenten der Einwirkungen $G$ und $H$ in Richtung der Ebene berechnet werden: $E=G \sin \beta+$ $H \cos \beta$. Mit dem maximal mobilisierbaren Widerstand aus der Normalkraft zur Ebene $R=$ $N \tan \varphi=(G \cos \beta-H \sin \beta) \tan \varphi$ kann die Sicherheit $\mathrm{zu}$

$$
\eta=\frac{R}{E}=\frac{\tan \varphi-\frac{H}{G} \tan \beta \tan \varphi}{\tan \beta+\frac{H}{G}}
$$

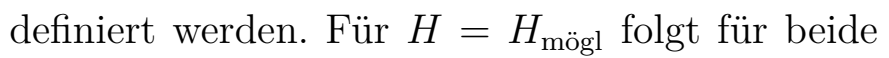
Sicherheitsdefinitionen (16) und (17) $\eta=\eta_{H}=1$ (Bild 18), also scheinen beide Definitionen vernünftig.

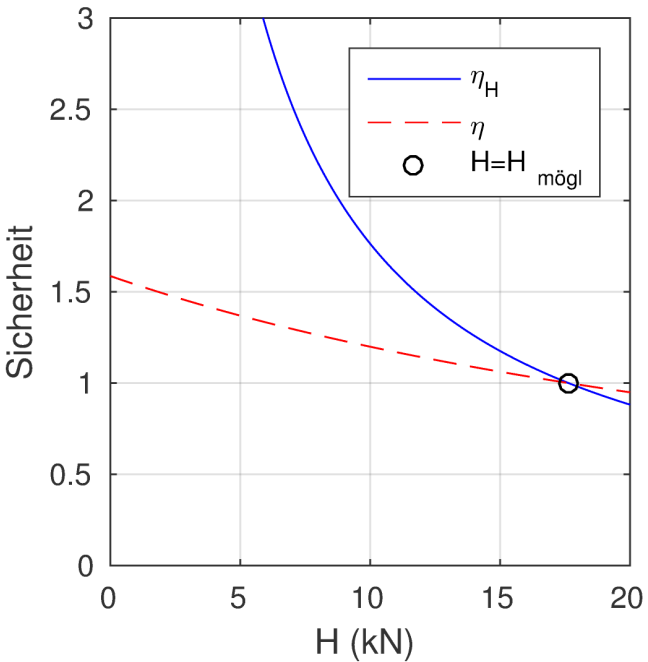

Bild 18: Werte für die Sicherheiten $\eta$ und $\eta_{H}$ des Blockes in Bild 16 $\left(G=100 \mathrm{kN} / \mathrm{m}^{2}\right.$, $\left.\beta=20^{\circ}, \varphi=30^{\circ}\right)$ nach den Definitionen (17) und (16) in Abhängigkeit von der Horizontalkraft $H$. Im Grenzzustand $H=H_{\text {mögl }}$ gilt $\eta=\eta_{H}=1$.

Es ist allerdings sofort ersichtlich, dass die Sicherheitsdefinition (16) für den Grenzfall $H \rightarrow 0$ gegen unendlich geht während die Definition (17) die Sicherheit des unbelasteten Blocks (14) liefert, was auch mechanisch konsistent ist. Wenn für das Beispiel in Bild 18 eine Sicherheit von 1,5 gefordert würde, dann wäre nach der Sicherheitsdefinition über die Horizontalkraft (16) $H=11,8 \mathrm{kN}$ zulässig, nach der Sicherheitsdefinition über die Beanspruchung und den Widerstand in der Scherfuge (17) nur $H=1,8 \mathrm{kN}$. Mechanisch ist für das Versagen die Ausnutzung der Reibungskraft in der Scherfuge maßgebend. Die Sicherheitsdefinition über eine mögliche Horizontalkraft führt zu einer wesentlich größeren Ausnutzung des Widerstandes in der Scherfuge!

Abschließend betrachten wir den Einfluss einer zusätzlichen Vertikalkraft auf die Sicherheit. Diese Frage taucht bei der Standischerheit in der tiefen Gleitfuge auf, wenn beurteilt werden soll, ob eine Verkehrslast günstig oder ungünstig wirkt. Betrachten wir zunächst den oben abgehandelten Fall mit einer ziehenden Horizontalkraft, die den Block ohne $P$ gerade ins Grenzgleichgewicht bringt, d.h es gilt $E=R$, Bild 19. Eine zusätzliche Vertikalkraft $P$ erhöht den Widerstand 
$R=N \tan \varphi$ mehr als die Einwirkung $E$. Sie wirkt also günstig, und zwar unabhängig von $\beta$.

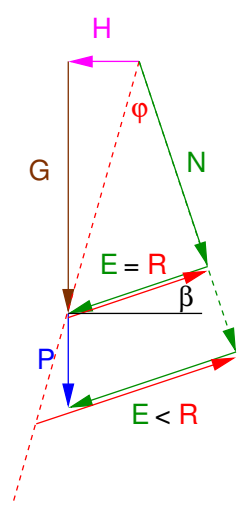

Bild 19: Einfluss einer zusätzlichen Vertikalkraft $P$ auf die Sicherheit bei einer destabilisierenden Horizontalkraft $H$.

Im Fall einer stützenden Horizontalkraft dreht sich dieser Effekt um, Bild 20. Hier erhöht $P$ die Einwirkung $E$ mehr als den Widerstand $R$ und wirkt somit ungünstig.

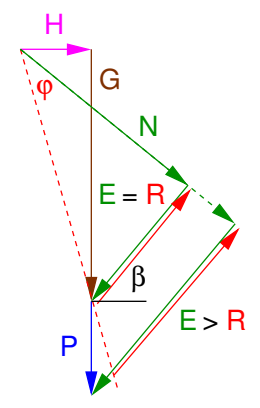

Bild 20: Einfluss einer zusätzlichen Vertikalkraft $P$ auf die Sicherheit bei einer stützen Horizontalkraft $H$.

Für $H=0$ hat $P$ sowie $G$ keinen Einfluss auf die Sicherheit. 